\title{
BMJ Open Circulating liver enzymes and risks of chronic diseases and mortality in the prospective EPIC-Heidelberg case- cohort study
}

\author{
Verena Katzke (D) , ${ }^{1}$ Theron Johnson, ${ }^{1}$ Disorn Sookthai, ${ }^{1}$ Anika Hüsing, ${ }^{1,2}$ \\ Tilman Kühn, ${ }^{1}$ Rudolf Kaaks ${ }^{1,2}$
}

To cite: Katzke V, Johnson T, Sookthai D, et al. Circulating liver enzymes and risks of chronic diseases and mortality in the prospective EPIC-Heidelberg casecohort study. BMJ Open 2020;10:e033532. doi:10.1136/ bmjopen-2019-033532

- Prepublication history and additional material for this paper are available online. To view these files, please visit the journal online (http://dx.doi. org/10.1136/bmjopen-2019033532).

Received 09 August 2019 Revised 24 January 2020 Accepted 27 January 2020

Check for updates

(C) Author(s) (or their employer(s)) 2020. Re-use permitted under CC BY-NC. No commercial re-use. See rights and permissions. Published by BMJ.

${ }^{1}$ Division of Cancer Epidemiology, Deutsches Krebsforschungszentrum, Heidelberg, Germany

${ }^{2}$ German Center for Lung

Research (DZL), Translational

Lung Research Center

Heidelberg, Heidelberg, Germany

Correspondence to

Dr Verena Katzke;

v.katzke@dkfz.de

\section{ABSTRACT}

Objectives Elevated liver enzyme concentrations in blood are indicative of liver diseases and may provide an early signal for being at risk for other chronic diseases. Our study aimed to assess the relationships of alkaline phosphatase (ALP), gamma-glutamyltransferase (GGT), alanine aminotransferase (ALT), aspartate transaminase (AST) and the De Ritis ratio (AST/ALT) with incidence and mortality of cardiovascular diseases (CVD) and the four most common cancers, that is, breast, prostate, colorectal and lung.

Setting, participants and outcome measures We analysed a case-cohort sample of the prospective EuropeanProspective Investigation into Cancer and Nutrition-Heidelberg cohort, including cancer $(n=1632)$, cancer mortality ( $n=761)$, CVD $(n=1070)$, CVD mortality $(\mathrm{n}=381)$ and a random subcohort $(\mathrm{n}=2739)$ with an average follow-up duration of 15.6 years. Concentrations of liver enzymes were measured in prediagnostic blood samples and Prentice-weighted Cox regression models were used to estimate HRs with $95 \%$ Cls.

Results High ALP levels were associated with increased risk for lung cancer and all-cause mortality (highest vs lowest quartile, multivariable adjusted $\mathrm{HR}=2.39(95 \% \mathrm{Cl}$ 1.30 to 4.39$), \mathrm{HR}=1.31$ (95\% $\mathrm{Cl} 1.02$ to 1.67$)$ ), high AST levels with all-cause mortality $(\mathrm{HR}=1.45(95 \% \mathrm{Cl} 1.15$ to 1.82)), and a high De Ritis ratio with prostate cancer risk, all-cause and cancer mortality ( $\mathrm{HR}=1.61(95 \% \mathrm{Cl} 1.10$ to 2.36$), \mathrm{HR}=1.60$ (95\% $\mathrm{Cl} 1.25$ to 2.04$), \mathrm{HR}=1.67$ (95\% Cl 1.26 to 2.23)). Using cut-points for liver enzyme levels above normal, we observed positive associations for allcause mortality with ALP, GGT and AST, and assigning a combined risk score resulted in positive associations with all-cause and cause-specific mortality.

Conclusions Measurements of serum liver enzymes, as routinely performed in health check-ups, may support the identification of individuals at increased risk for all-cause mortality. Further prospective studies are needed to verify our first results on individual cancers and on a combined risk score.

\section{BACKGROUND}

The four liver enzymes alkaline phosphatase (ALP), gamma-glutamyltransferase (GGT), alanine aminotransferase (ALT) and
Strengths and limitations of this study

Major chronic diseases and mortalities investigated in one comprehensive study.

- Large sample size allowed subgroup analyses and control for multiple confounders, including lifestyle information and further biomarker and exclusion of participants with self-reported prevalent liver insufficiency in sensitivity analyses.

- Prospective design limits reverse causation bias.

- Potential of residual confounding due to measurement error or unmeasured confounders.

aspartate transaminase (AST) are commonly measured in clinical context to determine liver disease, as they are released into the blood stream after liver injury. ${ }^{1}$ Because AST has a relatively short half-life compared with ALT (18hours vs 36 hours), ALT increases more than AST soon after liver injury and, thus, the AST over ALT ratio (referred as 'De Ritis ratio') represents the time course and aggressiveness of disease in most circumstances. ${ }^{2}$ Because assays for these enzymes are inexpensive and routinely applied in clinical laboratory tests, they may be useful for the rapid identification of individuals at high risk for certain diseases. Indeed, a growing body of epidemiological evidence indicates that blood levels of liver enzymes are associated with liver injuries and with a wider range of disease outcomes, including type 2 diabetes mellitus, ${ }^{3}{ }^{4}$ non-alcoholic fatty liver disease ${ }^{5}$ and cardiovascular diseases (CVD), ${ }^{6-10}$ as well as with all-cause and cause-specific mortality. ${ }^{11-14}$

However, previous prospective studies have predominantly focused on GGT and its associations with incident CVD and all-cause mortality, whereas ALP and the two transaminases have received less attention. ${ }^{71415}$ Moreover, evidence summarised in a meta-analysis 
indicates that increasing levels of GGT and ALT may also be related to incident cancer risk. ${ }^{16}$ However, the number of included studies for site-specific cancers was limited and only a few further prospective studies on GGT and cancer risks were conducted since then. ${ }^{17-19}$

As far as we are aware, no prospective study has been conducted to comprehensively evaluate the associations of ALT with risks of the most common cancer entities lung, prostate and breast, and no study so far has examined the associations of ALP and AST with any organspecific cancer entities. In addition, the AST/ALT ratio (De Ritis ratio) has been studied as a prognostic tool in patients with various diseases, but less so as a risk factor for mortality ${ }^{20}$ and not at all in relation to incident chronic diseases in general, including CVD.

In view of these information deficits, we comprehensively assessed the relationships of the four liver enzymes and the De Ritis ratio with risk of major chronic diseases (myocardial infarction (MI), stroke and cancers of the lung, colorectum, prostate and breast) and all-cause, CVD-related and cancer-related mortality within the prospective European Prospective Investigation into Cancer (EPIC)-Heidelberg cohort. This is the first investigation systematically covering all four liver enzymes in relation to all the mentioned endpoints within a single prospective study. We hypothesise that GGT increases risk of incident CVD, that GGT is positively associated with allcause mortality, and that GGT and ALT increase cancer risk; whereas association analyses of AST, ALT and the De Ritis ratio with incident cancer and mortality are of exploratory nature.

\section{METHODS}

\section{Study setting and design}

The current case-cohort study is embedded within the EPIC-Heidelberg cohort, which comprises 25546 men and women from the general population living in the southern German city Heidelberg and its surrounding municipality at recruitment. The study design and methods for the EPIC study have been described in detail previously. ${ }^{21}$ Briefly, this multicentre European cohort recruited men and women, aged between 35 and 70 years in 23 centres in 10 European countries between 1992 and 2000. Baseline examinations included measurement of anthropometric indices by trained staff, assessment of lifestyle factors and dietary habits via comprehensive questionnaires and collection of a blood sample. The latter was drawn at the day of recruitment, independent of the participants fasting status, and kept at $+4^{\circ} \mathrm{C}$ to $+10^{\circ} \mathrm{C}$ for a maximum of 24 hours until centrifugation and further processing. Blood was then aliquoted into fractions of plasma, serum, erythrocytes and buffy coat and half of the aliquots were stored in liquid nitrogen at $-190^{\circ} \mathrm{C}$ in the centres. Due to the applied procedures for long-term storage, intact blood cells used for blood cell counting are not available. Informed consent was obtained from all participants at baseline.

\section{Patient and public involvement}

Patients and/or members of public were not involved in the design, or conduct, or reporting, or dissemination of the research.

\section{Follow-up and outcome classification}

Participants in EPIC-Heidelberg were followed from study entry (1994-1998) until chronic disease diagnosis, death or end of the follow-up period. Outcomes of cancer incidence were based on regional cancer registry data, hospitalisation records or active follow-up of study subjects and their next of kin. The latter approach plus hospitalisation records were applied to ascertain incident MI and stroke cases. Mortality outcomes were ascertained from death certificates which were collected from mortality registries. For the case-cohort study, verified incident breast, colorectum, prostate and lung cancer cases up to the end of 2012 and verified incident MI and stroke cases and verified deceased subjects up to the end of 2009 were included. Exclusion criteria were the occurrence of other malignant tumours preceding the diagnosis of the respective cancer, except for non-melanoma skin cancer, and non-availability of blood specimens. We did not exclude participants with prevalent liver insufficiency a priori, because (1) only self-reported data were available and (2) from less than $90 \%$ of the participants. We included 627 cases of breast (International Classification of Diseases (ICD)-10: C50), 554 cases of prostate (ICD-10: C61), 195 cases of lung (ICD-10: C34) and 256 cases of colorectal cancer (ICD-10: C18-20). In addition, we included 555 cases of MI (ICD-10: I21) and 515 cases of stroke (ICD10: I60, I61, I63, I64; $73 \%$ of ischaemic type). Number of incident events in the EPIC cohort were comparable to the 10-year prevalence of the German population in a similar age range. Within the entire EPIC-Heidelberg cohort, 1516 deceased until end of follow-up (December 2009), of which 761 (50\%) died of cancer, 381 (25\%) of cardiovascular events and the remaining $374(25 \%)$ of miscellaneous conditions that were also coded according to the ICD-10. The four most common cancer deaths in our study were due to lung ( $\mathrm{n}=138$ cases out of $761,18 \%)$, colorectal $(\mathrm{n}=85,11 \%)$, breast $(\mathrm{n}=69,9 \%)$ and pancreas cancer $(n=60,8 \%)$. A randomly selected subcohort with available blood specimens of about $10 \%$ of the entire EPIC-Heidelberg cohort participants serves as the reference pool $(n=2739)$. Within a case cohort, cases within a cohort are selected up to a censoring date-likewise to a nested case-control study, but several types of cases can be selected. The comparison group, however, does not consist of matched control participants free of the disease of interest, but of a representative subsample of the full cohort-the so-called subcohort, which also includes incident cases proportional to the full cohort. ${ }^{22}$ We chose the well-established case-cohort design because several failure time outcomes can be analysed with the same comparison group, as the random sample subcohort is selected independent of the outcome, and to save precious samples and costs. 


\section{Laboratory methods}

The frozen serum samples were sent on dry ice to Scandinavian Health Ltd (SHL) laboratories (Etten-Leur, Netherlands) for basic clinical chemistry measurements, including total ALP, GGT, AST and ALT. Serum concentrations of the analytes were determined using the Roche Cobas 6000 analytical system for clinical chemistry, according to the manufacturer's protocols. Normal reference values according to SHL laboratories are $\leq 125 \mathrm{U} / \mathrm{L}$ for ALP, $\leq 38 \mathrm{U} / \mathrm{L}$ (women) and $\leq 55$ (men) for GGT, $\leq 31 \mathrm{U} / \mathrm{L}$ (women) and $\leq 35 \mathrm{U} / \mathrm{L}$ (men) for AST, and $\leq 34 \mathrm{U} / \mathrm{L}$ (women) and $\leq 45 \mathrm{U} / \mathrm{L}$ (men) for ALT. ${ }^{23}$ The De Ritis ratio was calculated as the ratio of AST over ALT. ${ }^{24}$ Missing enzymes were remeasured $(<1 \%$ of the study population).

\section{Statistical analyses}

We estimated HRs and 95\% CIs based on Prenticeweighted Cox proportional hazard regression models with age as the underlying time scale, a common approach to account for incident diseases of interest in the subcohort. ${ }^{25}$ All analyses were stratified by sex and integer values of age at recruitment. Proportional hazards assumption was not violated in any of the models according to an extended version of the Schoenfeld residuals test. ${ }^{26}$ Blood markers entered the models continuously log 2 transformed (to approximate normal distribution), in quartiles with sexspecific cut-points based on the distribution in the subcohort and the lowest quartile considered as the reference category, by predefined reference cut-points above versus within the range of normal values, or continuously as a multimarker score. The multimarker score was built by assigning each participant a value between 0 and 4 depending on the enzyme levels being below $(=0)$ or above $(=1)$ the reference cut-points and adding up the values to a final score.

Tests for linear trend over blood marker levels were based on the median of each quartile modelled as a continuous variable. The multivariable-adjusted model included body mass index (BMI), height, waist circumference, lifetime alcohol consumption, smoking history (never, former, current, years since quitting smoking, numbers of cigarettes smoked, age started smoking), education, physical activity and diabetes mellitus. Cox models with breast cancer as an outcome were further adjusted for oral contraceptive use, hormone replacement therapy, menopausal status and full-term pregnancies; and those with CVD for total and high-density lipoprotein cholesterol, triglycerides and $\mathrm{C}$ reactive protein. Additional adjustments for liver enzymes did not or only marginally change the results and they were therefore omitted as potential confounders. In exploratory analyses, potential effect modification by median alcohol consumption (above vs below the median, that is, $4 \mathrm{~g} / \mathrm{d}$ for women and $20 \mathrm{~g} / \mathrm{d}$ for men) was tested with $\mathrm{Q}$ statistics, adjusted for age at recruitment and sex. Mediators/ confounders were selected a priori based on known or suspected interactions/relationships with liver enzymes and/or outcomes; the variables were completely known with no missing data. To assess possible reverse causation bias, sensitivity analyses were conducted excluding the first 2 years of follow-up. Further sensitivity analyses were conducted while excluding those with self-reported prevalent liver insufficiency assessed during questionnairebased follow-up rounds ('Has your doctor ever diagnosed you with...', n=48, HR generally unchanged and, therefore, not shown) or heavy alcohol consumers at baseline, that is, $\geq 96 \mathrm{~g} / \mathrm{d}$ for men and $\geq 60 \mathrm{~g} / \mathrm{d}$ for women, because excess alcohol intake influences liver metabolism.

All statistical tests were two sided to account for unknown risk directions in exploratory analyses and $\mathrm{p}$ values less than 0.05 indicate statistical significance. All analyses were performed using SAS V.9.4 (SAS Institute).

\section{RESULTS}

Baseline characteristics of the study population are displayed in table 1. Briefly, median age of cases at diagnosis varied between 60 (breast cancer) and 66 years (prostate cancer and CVD death) and mean BMI was highest among later CVD deaths $\left(28.7 \mathrm{~kg} / \mathrm{m}^{2}\right)$. Women prospectively diagnosed with breast cancer were mostly never smokers at recruitment $(55 \%)$-as compared with women of the subcohort $(51 \%)$-whereas those diagnosed with lung cancer were predominantly current smokers $(67 \%)$. Consumption of alcohol was higher among men compared with women but no differences in consumption pattern were observed by individual endpoint compared with the general cohort. The highest prevalence of self-reported diabetes mellitus was observed in participants with later MI $(13 \%)$ and who died of CVD during follow-up (14\%). Median follow-up time of the subcohort was 15.6 years, of cancer incidence and mortality in the range of 7.6-9.4 and of CVD incidence and mortality in the range of 7.8-8.1 years.

Among subcohort participants, ALT levels significantly correlated with GGT and AST levels ( $r=0.53$ and $r=0.64$, respectively) and to a lesser extent GGT with AST levels ( $r=0.36$, figure 1). ALP levels correlated only weakly with the other liver enzymes and correlations with further blood biomarkers (ie, lipids, iron metabolites, platelet activation factors), dietary (ie, alcohol intake) or anthropometric variables were generally weak.

\section{Liver enzyme levels and incident cancers}

High circulating ALP levels were associated with an increased risk of incident lung cancer after multivariate adjustment (highest vs lowest quartile, Q4 vs Q1, HR 2.39 (95\% CI 1.30 to 4.39 ), table 2). Excluding participants with heavy alcohol intake $(\geq 96 \mathrm{~g} / \mathrm{d}$ for men and $\geq 60 \mathrm{~g} / \mathrm{d}$ for women) reduced the ALP-lung cancer association further to 1.97 (95\% CI 1.05 to 3.68$)$, but the HR remained statistically significant (data not in tables). The two transaminases AST and ALT were not associated with lung cancer risk. 


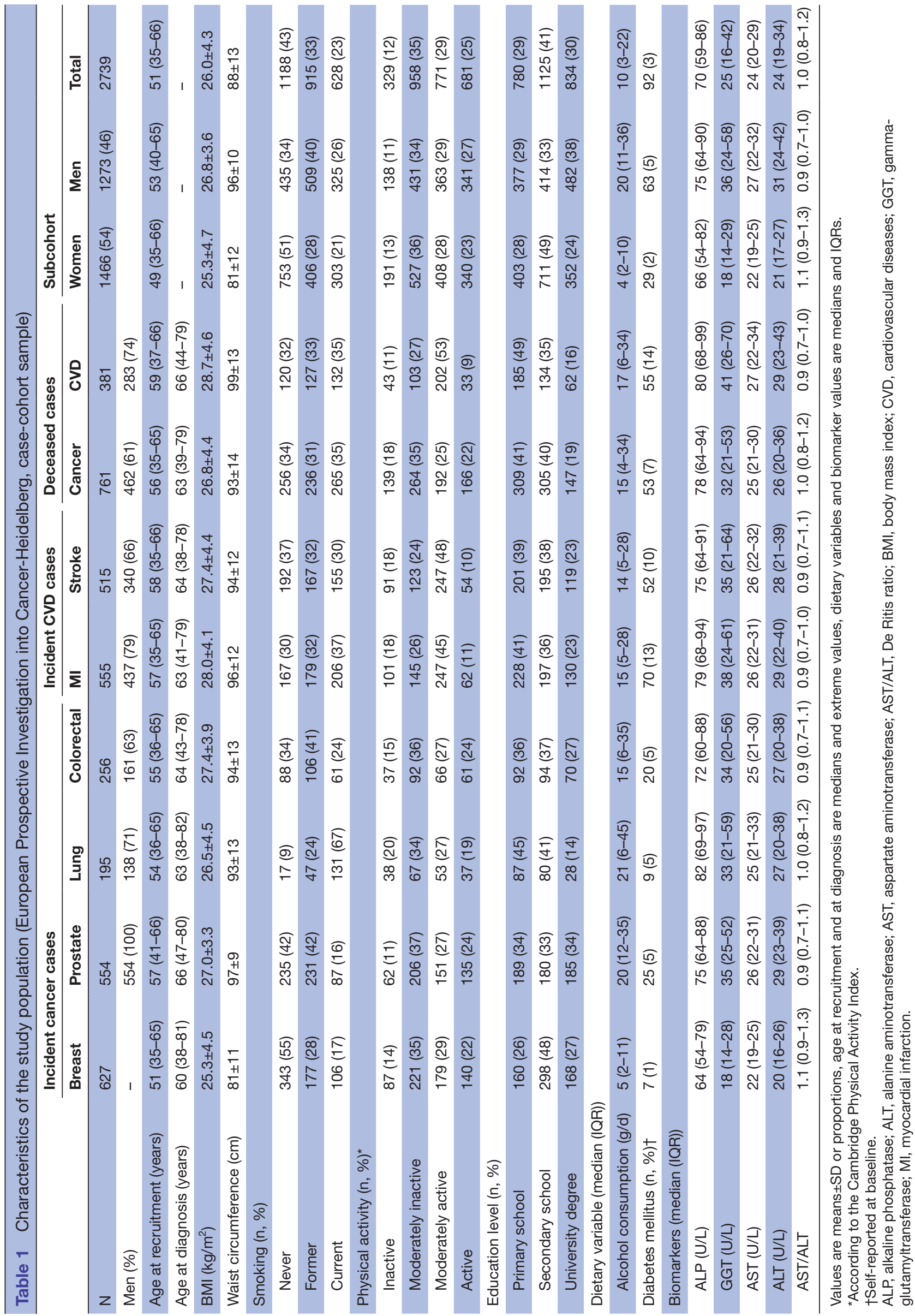




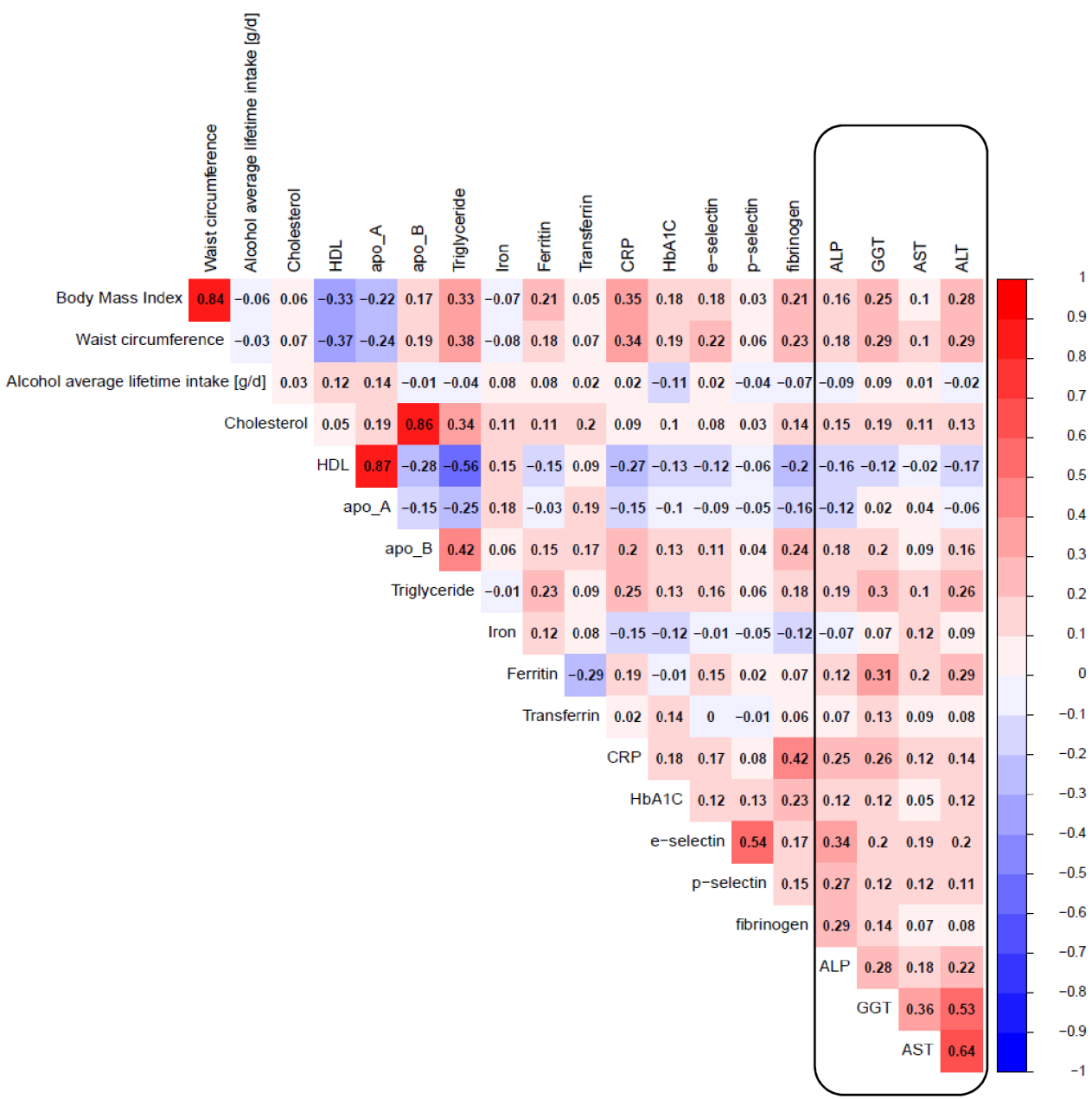

Figure 1 Spearman partial correlation of liver enzymes with covariates in the European Prospective Investigation into Cancer-Heidelberg subcohort $(n=2739)$, adjusted for sex and age at blood donation. Red depicts positive correlation and blue depicts negative correlation; the darker the shade, the stronger the correlation. ALP, alkaline phosphatase; ALT, alanine aminotransferase; apo, apolipoprotein; AST, aspartate aminotransferase; CRP, C reactive protein; GGT, gammaglutamyltransferase; HbA1c, glycosylated haemoglobin A1c; HDL, high-density lipoprotein; Lp(a), lipoprotein a; N, number of subjects; TG, triglycerides.

None of the enzymes were associated with risk of colorectal or breast cancer. A high De Ritis ratio was associated with a 1.6-fold increased risk of prostate cancer ( $Q 4$ vs Q1, HR 1.61 (95\% CI 1.10 to 2.36)), whereas single liver enzymes were not associated with risk (table 2).

\section{Liver enzyme levels and incident CVD}

None of the investigated liver enzymes were associated with incident MI or stroke after multivariate adjustments in our prospective case-cohort study (table 3). Subgroup analyses by stroke type (ischaemic vs haemorrhagic) did not reveal any significant associations with liver enzymes either, but number of participants with haemorrhagic stroke were limited to 90 out of 555 (data not shown).

\section{Liver enzymes and mortality}

Circulating ALP and AST levels in the highest quartile and a high De Ritis ratio were significantly associated with all-cause mortality after multivariable adjustment compared with the lowest quartiles $\left(\mathrm{HR}_{\mathrm{ALP}} 1.31\right.$ (95\% CI 1.02 to 1.67$), \mathrm{HR}_{\mathrm{AST}} 1.45$ (95\% CI 1.15 to 1.82 ), $\mathrm{HR}_{\text {deRitis }}$
1.60 (95\% CI 1.25 to 2.04), table 4). Excluding participants with heavy alcohol consumption attenuated the HR for all-cause mortality with high ALP levels to nonsignificance (data not in tables).

High AST levels and a high De Ritis ratio were also associated with cancer mortality in our case cohort $\left(\mathrm{HR}_{\mathrm{AST}}\right.$ 1.34 (95\% CI 1.02 to 1.74 ), $\mathrm{HR}_{\text {deRitis }} 1.67$ (95\% CI 1.26 to $2.23)$, table 4 ). The four most common causes of cancer death in our study were cancers of the lung ( $\mathrm{n}=138$ cases out of $761,18 \%)$, colorectum $(n=85,11 \%)$, breast $(n=69$, $9 \%)$ and pancreas $(\mathrm{n}=60,8 \%)$. In crude models, high ALP levels and a high De Ritis ratio were associated with lung $\left(\mathrm{HR}_{\mathrm{ALP}} 2.70\right.$ (95\% CI 1.43 to 5.11$), \mathrm{HR}_{\text {deRitis }} 2.25$ (95\% CI 1.24 to 4.07)), high ALT levels with breast (HR 2.13 (95\% CI 1.10 to 4.12)) and high AST levels with pancreatic cancer mortality (HR 2.31 (95\% CI 1.09 to 4.91 )). None of the liver enzymes were associated with colorectal cancer mortality (data not in tables).

Crude HRs for CVD mortality were significantly above one for all four enzymes comparing the highest to the 
Table 2 HRs and $95 \% \mathrm{Cl}$ for associations of circulating liver enzyme levels with incident cancers, crude and multivariable adjusted $^{*}$

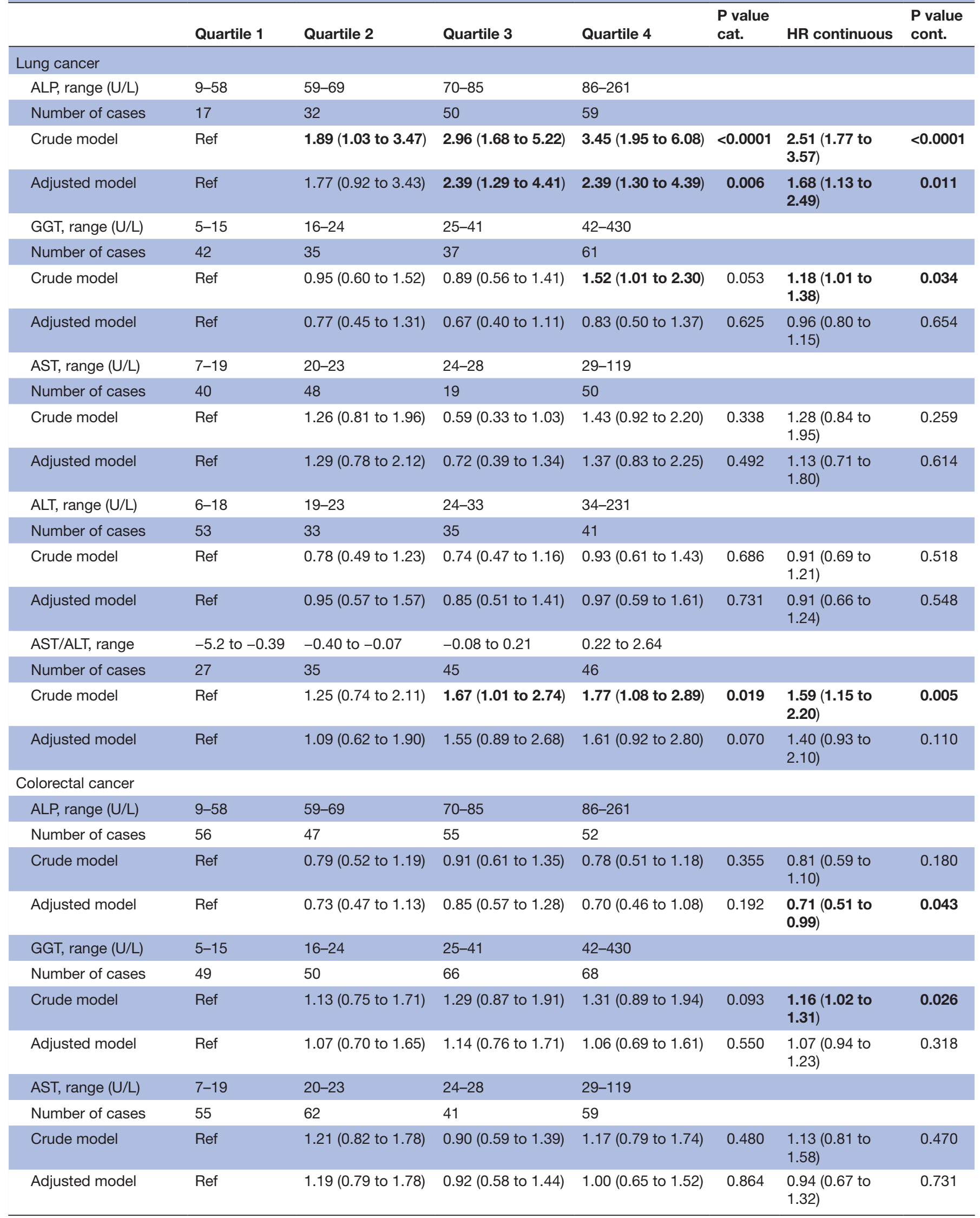


Table 2 Continued

\begin{tabular}{|c|c|c|c|c|c|c|c|}
\hline & Quartile 1 & Quartile 2 & Quartile 3 & Quartile 4 & $\begin{array}{l}P \text { value } \\
\text { cat. }\end{array}$ & HR continuous & $\begin{array}{l}\text { P value } \\
\text { cont. }\end{array}$ \\
\hline ALT, range $(\mathrm{U} / \mathrm{L})$ & $6-18$ & $19-23$ & $24-33$ & $34-231$ & & & \\
\hline Crude model & Ref & 0.83 (0.55 to 1.25$)$ & $1.08(0.74$ to 1.57$)$ & 0.92 (0.62 to 1.37$)$ & 0.797 & $\begin{array}{l}1.05 \text { (0.84 to } \\
1.32)\end{array}$ & 0.666 \\
\hline Adjusted model & Ref & 0.81 (0.53 to 1.23$)$ & $0.94(0.63$ to 1.41$)$ & 0.69 (0.45 to 1.07$)$ & 0.237 & $\begin{array}{l}0.89 \text { (0.70 to } \\
1.13)\end{array}$ & 0.339 \\
\hline Number of cases & 46 & 62 & 56 & 48 & & & \\
\hline Crude model & Ref & 1.27 (0.85 to 1.90$)$ & 1.23 (0.81 to 1.86$)$ & 1.11 (0.72 to 1.70$)$ & 0.660 & $\begin{array}{l}0.99(0.73 \text { to } \\
1.34)\end{array}$ & 0.933 \\
\hline Adjusted model & Ref & 1.34 (0.88 to 2.04$)$ & 1.45 (0.94 to 2.24$)$ & 1.35 (0.86 to 2.12$)$ & 0.149 & $\begin{array}{l}1.13(0.82 \text { to } \\
1.55)\end{array}$ & 0.456 \\
\hline Crude model & Ref & 1.01 (0.73 to 1.41$)$ & 1.06 (0.76 to 1.48$)$ & 0.88 (0.63 to 1.24$)$ & 0.486 & $\begin{array}{l}0.90 \text { (0.68 to } \\
1.20)\end{array}$ & 0.473 \\
\hline Adjusted model & Ref & $1.02(0.72$ to 1.44$)$ & $1.09(0.77$ to 1.54$)$ & 0.91 (0.64 to 1.30$)$ & 0.649 & $\begin{array}{l}0.93 \text { (0.69 to } \\
1.26)\end{array}$ & 0.648 \\
\hline GGT, range (U/L) & $5-15$ & $16-24$ & $25-41$ & $42-430$ & & & \\
\hline Number of cases & 122 & 142 & 130 & 101 & & & \\
\hline Crude model & Ref & $1.29(0.94$ to 1.77$)$ & $1.13(0.83$ to 1.56$)$ & 0.89 (0.64 to 1.24$)$ & 0.162 & $\begin{array}{l}0.94 \text { (0.84 to } \\
1.06)\end{array}$ & 0.295 \\
\hline Adjusted model & Ref & 1.40 (1.00 to 1.96$)$ & $1.22(0.87$ to 1.72$)$ & 1.00 (0.69 to 1.44$)$ & 0.394 & $\begin{array}{l}0.98 \text { ( } 0.86 \text { to } \\
1.11)\end{array}$ & 0.705 \\
\hline ALT, range (U/L) & $6-18$ & $19-23$ & $24-33$ & $34-231$ & & & \\
\hline Number of cases & 153 & 113 & 110 & 96 & & & \\
\hline Crude model & Ref & 0.85 (0.61 to 1.16$)$ & 0.81 (0.59 to 1.11$)$ & $0.83(0.60$ to 1.16$)$ & 0.322 & $\begin{array}{l}0.89 \text { (0.73 to } \\
1.07)\end{array}$ & 0.212 \\
\hline Adjusted model & Ref & 0.85 (0.61 to 1.18$)$ & 0.80 (0.57 to 1.13$)$ & 0.84 (0.59 to 1.19$)$ & 0.377 & $\begin{array}{l}0.90(0.73 \text { to } \\
1.11)\end{array}$ & 0.322 \\
\hline AST/ALT, range & -5.2 to -0.39 & -0.40 to -0.07 & -0.08 to 0.21 & 0.22 to 2.64 & & & \\
\hline Number of cases & 83 & 125 & 100 & 146 & & & \\
\hline Crude model & Ref & 1.28 (0.90 to 1.83$)$ & 0.96 (0.67 to 1.38$)$ & 1.54 (1.09 to 2.19$)$ & 0.038 & $\begin{array}{l}1.19 \text { (0.92 to } \\
1.54)\end{array}$ & 0.194 \\
\hline Adjusted model & Ref & 1.40 (0.96 to 2.04$)$ & 1.00 (0.68 to 1.47$)$ & $1.61(1.10$ to 2.36$)$ & 0.048 & $\begin{array}{l}1.19 \text { (0.90 to } \\
1.57)\end{array}$ & 0.231 \\
\hline \multicolumn{8}{|l|}{ Breast cancer† } \\
\hline ALP, range (U/L) & $9-58$ & $59-69$ & $70-85$ & $86-261$ & & & \\
\hline Number of cases & 141 & 146 & 121 & 112 & & & \\
\hline Crude model & Ref & 0.95 (0.72 to 1.27$)$ & 0.78 (0.58 to 1.05$)$ & $0.68(0.49$ to 0.93$)$ & 0.007 & $\begin{array}{l}0.76(0.61 \text { to } \\
0.95)\end{array}$ & 0.016 \\
\hline Adjusted model & Ref & $1.10(0.46$ to 2.61$)$ & 1.27 (0.52 to 3.10$)$ & 0.93 (0.34 to 2.55$)$ & 0.782 & $\begin{array}{l}0.93(0.44 \text { to } \\
1.96)\end{array}$ & 0.854 \\
\hline GGT, range (U/L) & $5-15$ & $16-24$ & $25-41$ & $42-430$ & & & \\
\hline
\end{tabular}




\begin{tabular}{|c|c|c|c|c|c|c|c|}
\hline & Quartile 1 & Quartile 2 & Quartile 3 & Quartile 4 & $\begin{array}{l}P \text { value } \\
\text { cat. }\end{array}$ & HR continuous & $\begin{array}{l}P \text { value } \\
\text { cont. }\end{array}$ \\
\hline Crude model & Ref & 0.87 (0.66 to 1.15$)$ & $0.74(0.56$ to 0.98$)$ & 0.79 (0.59 to 1.05$)$ & 0.198 & $\begin{array}{l}0.91 \text { ( } 0.81 \text { to } \\
1.03)\end{array}$ & 0.141 \\
\hline Adjusted model & Ref & 0.89 (0.66 to 1.19$)$ & 0.75 (0.56 to 1.00$)$ & 0.86 (0.63 to 1.18$)$ & 0.532 & $\begin{array}{l}0.95 \text { (0.84 to } \\
1.08)\end{array}$ & 0.467 \\
\hline AST, range (U/L) & $7-19$ & $20-23$ & $24-28$ & $29-119$ & & & \\
\hline Number of cases & 165 & 145 & 93 & 127 & & & \\
\hline Crude model & Ref & 1.09 (0.83 to 1.43$)$ & 0.74 (0.55 to 1.01$)$ & 0.87 (0.66 to 1.16$)$ & 0.154 & $\begin{array}{l}0.77 \text { (0.58 to } \\
1.01)\end{array}$ & 0.056 \\
\hline Adjusted model & Ref & 1.14 (0.85 to 1.52$)$ & 0.80 (0.59 to 1.10$)$ & 0.92 (0.68 to 1.24$)$ & 0.335 & $\begin{array}{l}0.81 \text { ( } 0.61 \text { to } \\
1.08)\end{array}$ & 0.154 \\
\hline Crude model & Ref & 1.04 (0.79 to 1.36$)$ & 0.83 (0.62 to 1.10$)$ & 0.82 (0.62 to 1.09$)$ & 0.111 & $\begin{array}{l}0.81 \text { ( } 0.69 \text { to } \\
0.97)\end{array}$ & 0.018 \\
\hline Adjusted model & Ref & 1.04 (0.78 to 1.38$)$ & 0.84 (0.62 to 1.13$)$ & 0.91 (0.67 to 1.23 ) & 0.420 & $\begin{array}{l}0.85 \text { ( } 0.71 \text { to } \\
1.02)\end{array}$ & 0.084 \\
\hline AST/ALT, range & -5.2 to -0.39 & -0.40 to -0.07 & -0.08 to 0.21 & 0.22 to 2.64 & & & \\
\hline Number of cases & 131 & 132 & 127 & 138 & & & \\
\hline Crude model & Ref & 0.93 (0.70 to 1.24$)$ & 1.06 (0.79 to 1.43$)$ & 1.17 (0.87 to 1.57$)$ & 0.205 & $\begin{array}{l}1.16 \text { (0.92 to } \\
1.47)\end{array}$ & 0.197 \\
\hline Adjusted model & Ref & 0.90 (0.67 to 1.22$)$ & 1.04 (0.76 to 1.42$)$ & 1.11 (0.81 to 1.52$)$ & 0.394 & $\begin{array}{l}1.11 \text { ( } 0.87 \text { to } \\
1.42)\end{array}$ & 0.393 \\
\hline
\end{tabular}

The values given in bold are significant when $p<0.05$.

${ }^{*}$ Crude model adjusted for sex and age at blood draw. Multivariable model additionally adjusted for baseline height, waist, body mass index, lifetime alcohol consumption, smoking history, education, diabetes and physical activity.

†Additionally adjusted for oral contraceptive use, hormone replacement therapy, menopausal status and full-term pregnancies. Quartile ranges are sex specific and based on the distribution in the subcohort.

ALP, alkaline phosphatase; ALT, alanine aminotransferase; AST, aspartate aminotransferase; AST/ALT, De Ritis ratio; GGT, gammaglutamyltransferase; HR continuous, continuous HR for a doubling in biomarker concentration; $p$-value cat, p-trend over lipid biomarker levels based on the median of each quartile category; p-value cont., p-trend continuously.

lowest quartile, but multivariable adjustment led to allcause non-significant associations. The De Ritis ratio was not associated with CVD mortality (table 4).

\section{Effect modification by alcohol consumption (data not in tables)}

Among participants drinking more than $21.5 \mathrm{~g} / \mathrm{d}$ or $4.5 \mathrm{~g} / \mathrm{d}$ alcohol (median intake of men or women, respectively), a high De Ritis ratio was significantly associated with an increased risk of all-cause death (continuous, adjusted HR 1.49 (95\% CI 1.14 to 1.96), p interaction 0.045). Associations with mortality among participants drinking less alcohol were not significant (continuous, adjusted HR 1.19 (95\% CI 0.91 to 1.56)). Further interaction analyses by median alcohol intake for other exposures or endpoints were not significant.

\section{Exclusion of first 2 years of follow-up (data not in tables)}

In sensitivity analyses, we excluded cases diagnosed within 2 years after baseline assessment, that is, 144 with incident cancer (16 lung, 27 colorectum, 37 prostate, 64 breast), 49 with incident MI, 46 with incident stroke and 125 deceased subjects (59 cancer, 37 CVD, 29 other) and left censored the remaining participants by 2 years of follow-up time. High ALP levels were no longer associated with an increased risk of lung cancer and all-cause mortality in the fully adjusted model (Q4 vs Q1, adjusted $\mathrm{HR}_{\text {lung }} 1.92$ (95\% CI 0.98 to 3.78$), \mathrm{HR}_{\text {death }} 1.23$ (95\% CI 0.95 to 1.60)). Similarly, high AST levels were not associated with cancer mortality any longer in the fully adjusted model (HR 1.27 (95\% CI 0.96 to 1.69)). Other associations were not influenced by these sensitivity analyses.

\section{Using predefined clinical cut-points and a multimarker score (supplementary tables)}

Between 3\% (ALP) and 20\% (GGT) of our subcohort participants had enzyme levels above the normal range. Participants above normal enzyme levels were at increased risk for all-cause mortality $\left(\mathrm{HR}_{\mathrm{ALP}} 1.67\right.$ (95\% CI 1.13 to 2.47 ), $\mathrm{HR}_{\mathrm{GGT}} 1.28$ (95\% CI 1.07 to 1.54 ), $\mathrm{HR}_{\mathrm{AST}} 1.39$ (95\% CI 1.10 to 1.75$)$ ) compared with those with normal levels after multivariable adjustments (online supplementary table 1). Combining the four liver enzymes into a risk score, where each participant was assigned a score between 0 and 4 depending on the enzyme levels being 
Table $3 \mathrm{HRs}$ and $95 \% \mathrm{Cl}$ for associations of circulating liver enzyme levels with incident cardiovascular diseases, crude and multivariable adjusted*

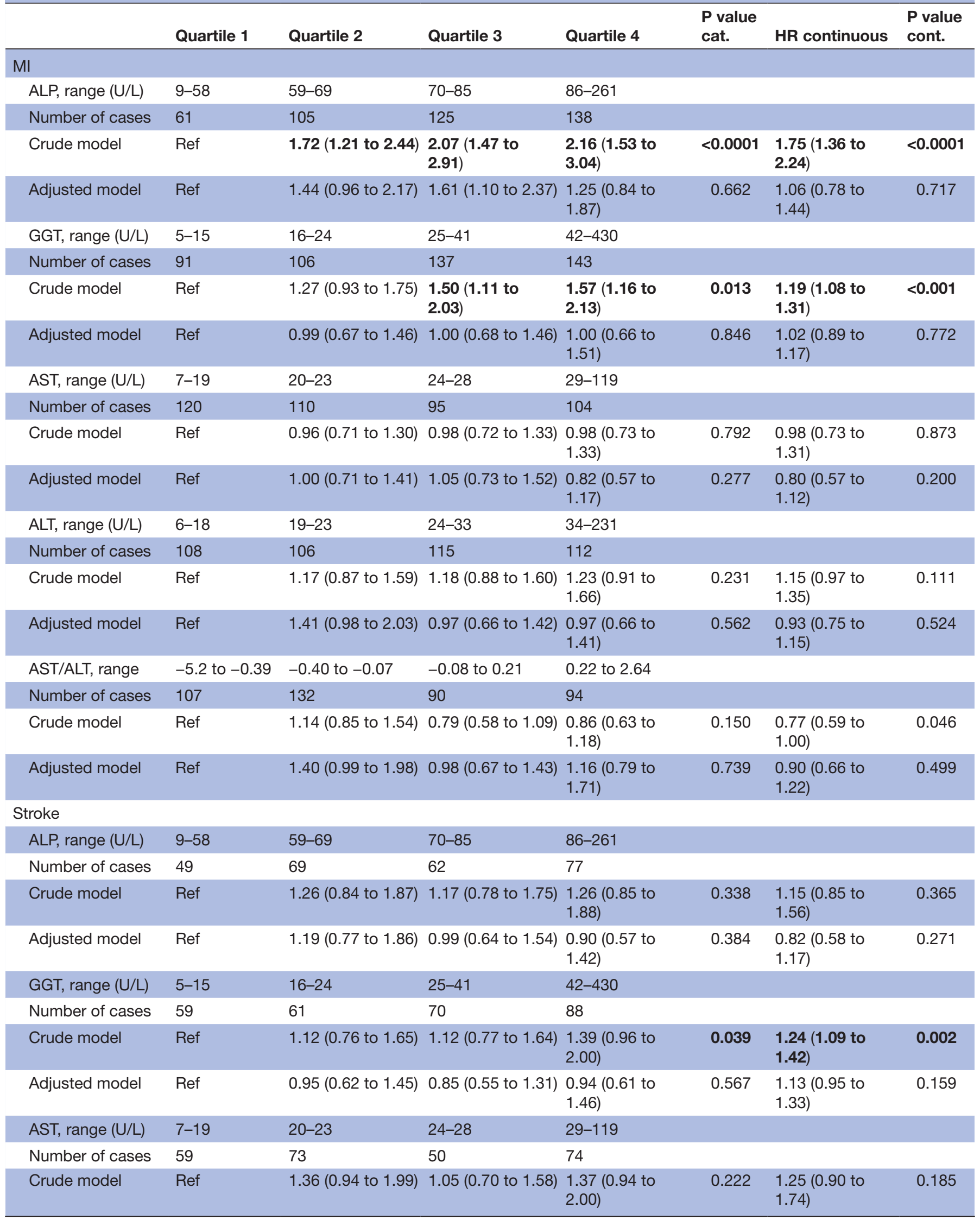




\begin{tabular}{|c|c|c|c|c|c|c|c|}
\hline & Quartile 1 & Quartile 2 & Quartile 3 & Quartile 4 & $\begin{array}{l}P \text { value } \\
\text { cat. }\end{array}$ & HR continuous & $\begin{array}{l}P \text { value } \\
\text { cont. }\end{array}$ \\
\hline ALT, range (U/L) & $6-18$ & $19-23$ & $24-33$ & $34-231$ & & & \\
\hline Adjusted model & Ref & 1.38 (0.91 to 2.08$)$ & 0.97 (0.63 to 1.49$)$ & $\begin{array}{l}0.95 \text { (0.63 to } \\
1.45)\end{array}$ & 0.514 & $\begin{array}{l}1.01(0.80 \text { to } \\
1.27)\end{array}$ & 0.939 \\
\hline AST/ALT, range & -5.2 to -0.39 & -0.40 to -0.07 & -0.08 to 0.21 & 0.22 to 2.64 & & & \\
\hline Number of cases & 62 & 70 & 56 & 67 & & & \\
\hline
\end{tabular}

The values given in bold are significant when $p<0.05$.

${ }^{*}$ Crude model adjusted for sex and age at blood draw. Multivariable model additionally adjusted for baseline height, waist, body mass index, lifetime alcohol consumption, smoking history, education, diabetes, physical activity, total and high-density lipoprotein cholesterol, triglycerides and $\mathrm{C}$ reactive protein. Quartile ranges are sex specific and based on the distribution in the subcohort.

ALP, alkaline phosphatase; AST, aspartate aminotransferase; AST/ALT, De Ritis ratio; GGT, gamma-glutamyltransferase; HR continuous, continuous HR for a doubling in biomarker concentration; MI, myocardial infarction; $p$-value cat, $p$-trend over lipid biomarker levels based on the median of each quartile category; p-value cont, p-trend continuously.

below (0) or above (1) the clinical cut-points, resulted in increased risks for all-cause, cancer and CVD mortality for a higher score (adjusted continuous HR per unit increase in the score, all-cause mortality $=1.16$ (95\% CI 1.06 to 1.27 , cancer mortality $=1.19$ (95\% CI 1.03 to 1.37 ), CVD mortality $=1.17$ (95\% CI 1.01 to 1.37 ), online supplementary table 2).

\section{DISCUSSION}

Elevated liver enzyme concentrations in blood are indicative of liver diseases and may provide an early signal for being at risk for other chronic diseases and mortality. In this well-characterised case-cohort study, we found that higher liver enzyme levels were associated with increased all-cause mortality and selectively also with cause-specific mortality and incident cancers, although associations varied for specific enzymes and disease endpoints. Using predefined clinical cut-points for liver enzyme levels above the normal range, we observed significant associations of GGT, ALP and AST with all-cause mortality, while a higher multimarker score resulted in positive associations with all-cause and cause-specific mortality. Further, we observed heterogeneity in the association high De Ritis ratio with mortality by alcohol drinking habits in exploratory analyses.

\section{Liver enzymes and CVD incidence and mortality}

Despite some evidence from previous investigations, we did not observe any association of elevated liver enzymes with risk of incidence or mortality from major cardiovascular events. However, our results are in line with recent evidence for GGT as a potential useful indicator for all-cause mortality, considering circulating levels above the normal threshold (ie, $>38 \mathrm{U} / \mathrm{L}$ for women and $>55$ for men in our study). ${ }^{13}$ Similarly, our data suggest that abovenormal levels of ALP and AST may also be relevant risk indicators for all-cause mortality. These findings are largely in line with results from previous studies covering one or more of the liver enzymes and all-cause mortality. ${ }^{10} 132728$ However, we were not able to observe further results found by others, such as associations of GGT and ALP with risk of CVD ${ }^{6}$ GGT, ALT and AST with incident MI or ischaemic stroke, ${ }^{7}$ ALP with CVD events and CVD mortality ${ }^{10}$ or ALT with all-cause mortality. ${ }^{27}$ A meta-analysis covering the four enzymes and their associations with all-cause mortality found significant positive risk estimates for GGT and ALP, as in our investigation, but opposing risks for ALT and no associations for AST. ${ }^{13}$ Further, a recent evaluation by Kunutsor and colleagues suggested even an inverse association between the two transaminases AST and ALT and total incident CVD in the Dutch Prevention of Renal and Vascular End-stage Disease (PREVEND) study. ${ }^{9}$ Inverse associations were also observed in a meta-analysis on the associations of ALT and all-cause, cancer and CVD mortality, but with potential heterogeneity by age such that associations among younger subjects (ie, <70) tended to be above whereas associations among older subjects (ie, $\geq 70$ ) tended to be below one. ${ }^{14}$ Unfortunately, we could not replicate Kunutsor's analyses using the 70-year age cutpoint, because of limited case numbers above age 70 . 
Table 4 HRs and $95 \% \mathrm{Cl}$ for associations of circulating liver enzyme levels with all-cause, cancer and cardiovascular diseases (CVD) mortality, crude and multivariable adjusted ${ }^{\star}$

\begin{tabular}{|c|c|c|c|c|c|c|c|}
\hline & Quartile 1 & Quartile 2 & Quartile 3 & Quartile 4 & $\begin{array}{l}P \text { value } \\
\text { cat. }\end{array}$ & HR continuous & $\begin{array}{l}P \text { value } \\
\text { cont. }\end{array}$ \\
\hline \multicolumn{8}{|l|}{ All-cause mortality } \\
\hline ALP, range (U/L) & $9-58$ & $59-69$ & $70-85$ & $86-261$ & & & \\
\hline Number of cases & 210 & 222 & 326 & 417 & & & \\
\hline Crude model & Ref & 0.99 (0.78 to 1.26$)$ & $1.46(1.16$ to 1.83$)$ & $1.66(1.33$ to 2.08$)$ & $<0.0001$ & $\begin{array}{l}1.83(1.50 \text { to } \\
2.23)\end{array}$ & $<0.0001$ \\
\hline GGT, range (U/L) & $5-15$ & $16-24$ & $25-41$ & $42-430$ & & & \\
\hline Number of cases & 250 & 259 & 291 & 467 & & & \\
\hline Crude model & Ref & $1.12(0.90$ to 1.40$)$ & 1.09 (0.87 to 1.35$)$ & $1.75(1.42$ to 2.15$)$ & $<0.0001$ & $\begin{array}{l}1.35(1.25 \text { to } \\
1.46)\end{array}$ & $<0.0001$ \\
\hline Crude model & Ref & $1.16(0.94$ to 1.44$)$ & 1.05 (0.84 to 1.32$)$ & $1.62(1.32$ to 2.00$)$ & $<0.0001$ & $\begin{array}{l}1.78 \text { (1.47 to } \\
2.15)\end{array}$ & $<0.0001$ \\
\hline Adjusted model & Ref & 1.11 (0.87 to 1.41$)$ & 1.12 (0.88 to 1.43$)$ & $1.45(1.15$ to 1.82$)$ & 0.003 & $\begin{array}{l}1.47 \text { (1.19 to } \\
1.82)\end{array}$ & $<0.001$ \\
\hline ALT, range $(\mathrm{U} / \mathrm{L})$ & $6-18$ & $19-23$ & $24-33$ & $34-231$ & & & \\
\hline Number of cases & 341 & 251 & 273 & 343 & & & \\
\hline Crude model & Ref & 0.87 (0.70 to 1.08$)$ & $0.86(0.70$ to 1.07$)$ & $1.18(0.96$ to 1.45$)$ & 0.105 & $\begin{array}{l}1.15 \text { (1.01 to } \\
1.30)\end{array}$ & 0.033 \\
\hline Adjusted model & Ref & 0.87 (0.69 to 1.10$)$ & 0.78 (0.61 to 0.99$)$ & $1.00(0.79$ to 1.26$)$ & 0.998 & $1.02(0.89$ to 1.18$)$ & 0.758 \\
\hline AST/ALT, range & -5.2 to -0.39 & -0.40 to -0.07 & -0.08 to 0.21 & 0.22 to 2.64 & & & \\
\hline ALP, range (U/L) & $9-58$ & $59-69$ & $70-85$ & $86-261$ & & & \\
\hline Number of cases & 115 & 116 & 176 & 198 & & & \\
\hline Crude model & Ref & 0.94 (0.70 to 1.26$)$ & $1.40(1.07$ to 1.84$)$ & 1.41 (1.08 to 1.86$)$ & 0.002 & $\begin{array}{l}1.53(1.21 \text { to } \\
1.93)\end{array}$ & $<0.001$ \\
\hline Adjusted model & Ref & 0.89 (0.65 to 1.21$)$ & 1.30 (0.98 to 1.73$)$ & 1.17 (0.88 to 1.56$)$ & 0.102 & $1.26(0.99$ to 1.61$)$ & 0.059 \\
\hline GGT, range (U/L) & $5-15$ & $16-24$ & $25-41$ & $42-430$ & & & \\
\hline Number of cases & 149 & 134 & 169 & 200 & & & \\
\hline Crude model & Ref & $0.98(0.75$ to 1.28$)$ & 1.05 (0.81 to 1.36$)$ & $1.24(0.97$ to 1.60$)$ & 0.069 & $\begin{array}{l}1.16 \text { (1.05 to } \\
1.27)\end{array}$ & 0.003 \\
\hline Adjusted model & Ref & 0.89 (0.67 to 1.18$)$ & 0.90 (0.68 to 1.17$)$ & 0.94 (0.71 to 1.23$)$ & 0.797 & $1.04(0.94$ to 1.16$)$ & 0.408 \\
\hline AST, range (U/L) & $7-19$ & $20-23$ & $24-28$ & $29-119$ & & & \\
\hline Number of cases & 146 & 162 & 112 & 191 & & & \\
\hline Crude model & Ref & 1.20 (0.93 to 1.56$)$ & 0.94 (0.71 to 1.25$)$ & 1.41 (1.10 to 1.81$)$ & 0.040 & $\begin{array}{l}1.35 \text { (1.08 to } \\
1.69)\end{array}$ & 0.009 \\
\hline Adjusted model & Ref & $1.18(0.90$ to 1.55$)$ & 0.99 (0.74 to 1.33$)$ & $1.34(1.02$ to 1.74$)$ & 0.126 & $1.21(0.95$ to 1.54$)$ & 0.122 \\
\hline ALT, range (U/L) & $6-18$ & $19-23$ & $24-33$ & $34-231$ & & & \\
\hline Number of cases & 196 & 133 & 137 & 154 & & & \\
\hline Crude model & Ref & 0.81 (0.63 to 1.05$)$ & 0.75 (0.58 to 0.97$)$ & 0.91 (0.71 to 1.16$)$ & 0.398 & $0.94(0.81$ to 1.10$)$ & 0.449 \\
\hline
\end{tabular}


Table 4 Continued

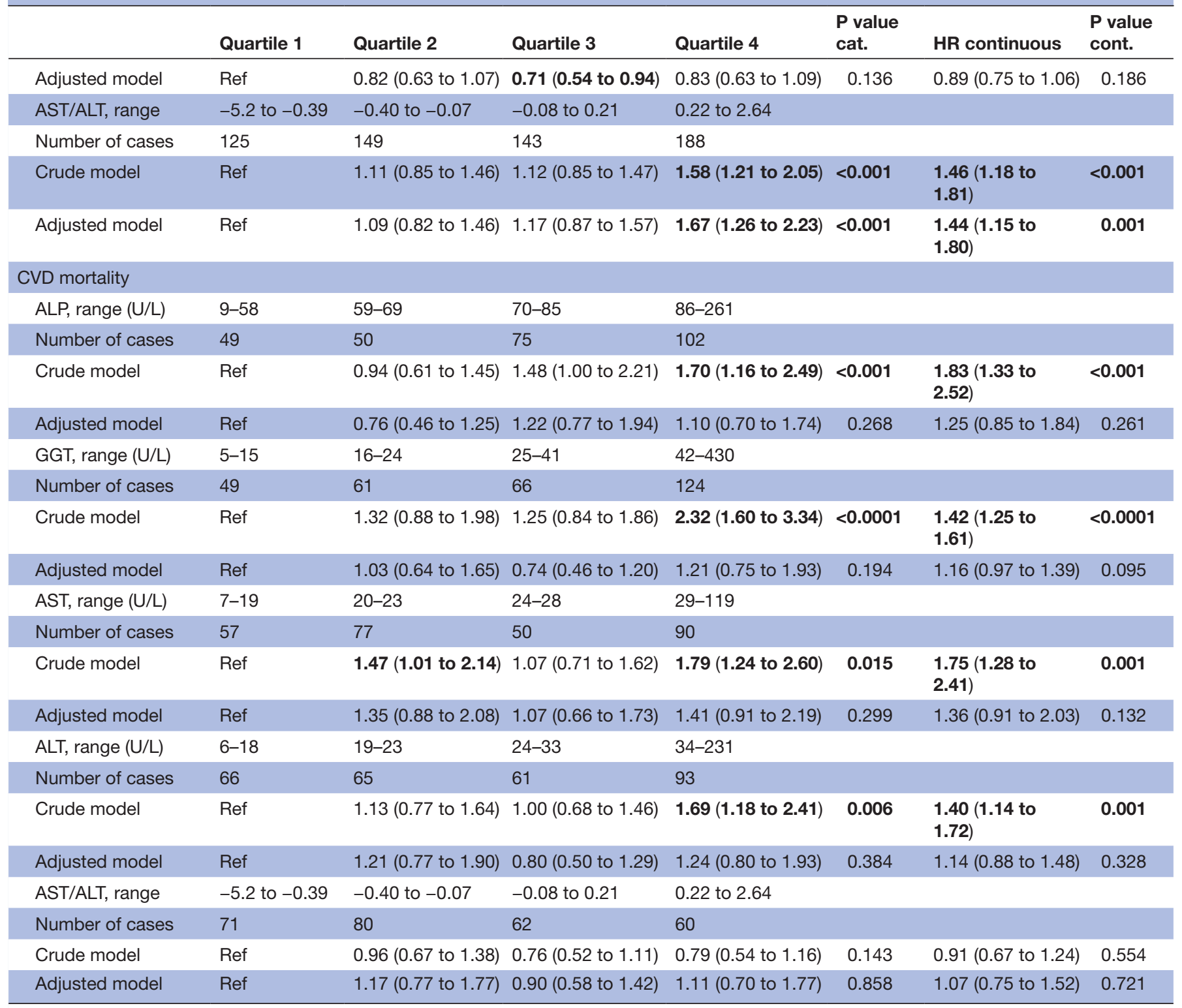

The values given in bold are significant when $p<0.05$.

${ }^{*}$ Crude model adjusted for sex and age at blood draw. Multivariable model additionally adjusted for baseline height, waist, body mass index, lifetime alcohol consumption, smoking history, education, diabetes and physical activity. Models with CVD death as an endpoint were additionally adjusted for total and high-density lipoprotein cholesterol, triglycerides and $\mathrm{C}$ reactive protein. Quartile ranges are sex specific and based on the distribution in the subcohort.

ALP, alkaline phosphatase; ALT, alanine aminotransferase; AST, aspartate aminotransferase; AST/ALT, De Ritis ratio; GGT, gammaglutamyltransferase; HR continuous, continuous HR for a doubling in biomarker concentration; $p$-value cat, $p$-trend over lipid biomarker levels based on the median of each quartile category; $\mathrm{p}$-value cont, $\mathrm{p}$-trend continuously.

Regarding the De Ritis ratio (AST/ALT), as a composite index of liver function, only one previous prospective investigation was conducted, in Japan, focusing exclusively on mortality endpoints. In this study, by Yokohama et al, which included a total of 250 deceased participants after 10 years of follow-up, a high AST/ALT ratio was found to be an independent predictor of all-cause and cardiovascular mortality. We observed a similar positive risk association with all-cause mortality and not with CVD mortality when multivariable adjustments were made, but with cancer mortality. Interaction analyses by median alcohol intake revealed that the association of a high De Ritis ratio with all-cause death was confined to participants with higher-than- average alcohol consumption, that is, $4 \mathrm{~g} / \mathrm{d}$ for women and $20 \mathrm{~g} / \mathrm{d}$ for men, which might be an indicator for advanced liver disease in these participants. According to Botros, the De Ritis ratio provides useful diagnostic and prognostic information ${ }^{2}$ and our results generally support the conclusion of Yokohoma that measuring this ratio during routine health check-ups may be a simple and cost-effective marker for all-cause, CVD and/or cancer mortality. 
While participants with above-normal values of liver enzymes had clearly increased risks for all-cause mortality, the associations of GGT were not apparent and those for ALP were weaker in analyses based on enzyme levels in quartile categories. In our categorical analyses, the top quartile category generally included high proportions of subjects with enzyme values that were elevated but largely within the normal range. And further, with each additional liver enzyme being above normal, the higher the risk for cancer, CVD and all-cause mortality. Especially for cause-specific mortality, a score based on all four liver enzymes is more informative than each enzyme alone. Thus, our observations indicate that enzyme values above the clinical normal range, as derived from assay-specific laboratory manuals and comparably to charts used by general practitioners for decision-making, and in combination may be predictive for risk of all-cause and cancer mortality. However, we also observed significant and strong associations of risks of incident lung cancer with more moderately elevated ALP levels, within the normal range (above $70 \mathrm{U} / \mathrm{L}$ and not only above $125 \mathrm{U} / \mathrm{L}$ ), suggesting that individuals with moderately increased ALP levels may deserve closer monitoring in view of risks of developing future lung cancer.

\section{Liver enzymes and cancer incidence and mortality}

Surprisingly, circulating liver enzyme levels were mostly not associated with risk of single incident cancers in our prospective study. Exceptions are the strong positive associations of ALP with lung cancer risk, as already depicted above, and positive associations of the De Ritis ratio with incident prostate cancer. While previous prospective investigations found rather consistent positive associations for high GGT levels with risk of lung, colorectal, prostate or breast cancer, limited (ALT and gastrointestinal cancers) or even zero studies of ALP, AST or the De Ritis ratio in relation to the four cancer entities have been conducted so far. Our subgroup analyses on median alcohol consumption should be treated with caution despite significant $\mathrm{p}$ values and HRs as number of cases within each subgroup were relatively small and are of exploratory nature. Because of the large number of tests performed, we cannot rule out that some of our observed associations are due to chance alone.

\section{Plausibility}

The most commonly proposed biological mechanisms for the increased risks of incident CVD and mortality with elevated levels of the four liver enzymes include the presence of underlying but unrecognised chronic liver diseases, which itself increases the risk of fatal and nonfatal CVD events. ${ }^{29}{ }^{30}$ Enzyme-specific rationales have also been postulated. For the associations of high GGT levels with risk of incident CVD, these include involvement of GGT in atherosclerosis by enhancing plaque formation, fostering an inflammatory milieu and triggering oxidative stress. ${ }^{31}$ Underlying pathways for the possible association of high GGT levels with incident lung cancer risk are unclear, but postulated mechanisms for GGT have been nicely summarised by Kunutsor and colleagues for overall cancer and include the persistent production of reactive oxygen species and hyperglycaemia. ${ }^{16}$ ALP is an enzyme that catalyses the hydrolysis of organic pyrophosphate, an inhibitor of vascular calcification, resulting in increased calcification of vessels ${ }^{32}$ - a well-documented risk factor for CVD. Why moderate increases in circulating ALP are also associated with higher lung cancer incidence is unclear. One possibility might be that high ALP levels appear to be independent predictors for bone metastases that are commonly detected in patients with lung cancer. $^{33} 34$ Thus, high ALP levels might be associated with lung cancer risk due to these unrecognised bone metastases years prior to a cancer diagnosis. Another possible explanation is residual confounding of smoking, which can never be ruled out with respect to lung cancer. Further studies are needed to confirm our findings on lung cancer, especially because these are based on the only prospective investigation so far.

\section{Strengths and limitations}

Our present analysis has several strengths. The large sample size allowed us to investigate associations with control for multiple confounders and in various subgroups. Further, the recruitment of disease-free individuals at baseline limits the possibility of reverse-causation bias as does the additional exclusion of participants with up to 2 years of follow-up in sensitivity analyse, where risks remained largely unchanged. We only observed a loss of significance in the associations of elevated ALP with lung cancer incidence and all-cause mortality as well as of elevated AST with cancer mortality. However, this might merely be due to a decline in case numbers, as the lower limit of the CIs are close to one and the risks are comparable in either model. Limitations of our investigation include the potential of residual confounding due to measurement error of potential confounders (ie, smoking and lung cancer) or even unmeasured confounders, such as family history of cancer, blood pressure or liver function tests. The latter were not possible to assess in our cohort, because quantification of blood cell counts necessary for score building is largely impossible in frozen buffy-coat pellets of blood lymphocytes (no storage of intact blood cells in our cohort). In addition, undetected liver diseases were only partly known by self-reporting and could, therefore, not be completely adjusted for. A further limitation is that blood was only taken once at baseline and might not reflect fluctuations of liver enzymes over time. However, liver enzymes are rather stable over time (for example, ALT does not fluctuate during the day ${ }^{35}$ ) and will only be elevated in response to a liver injury/disease. ${ }^{1}$ In the exploratory hypothesis generating analyses, we delved into detailed subgroup comparisons with sometimes reduced case numbers, and in the interest of providing a general overview and to point out findings of potential future interest, we report simple $\mathrm{p}$ values without further adjusting for multiple testing. We consider this more 
valuable than to overlook associations which may afford preventive potential at a low cost of potential verification (and application). Obtained results in subgroup analyses should be treated with caution as number of cases were relatively small and results reflect exploratory analyses.

\section{CONCLUSIONS}

Our results, based on a well-characterised population cohort and partly in line with findings from previous prospective investigations, indicate that measurements of serum liver enzymes, as routinely performed in health check-ups, may provide relevant indications for the identification of individuals at increased risk for all-cause mortality. However, our findings do not support a strong role of elevated liver enzymes with major CVD events or CVD mortality. Further prospective studies are needed to verify our first results on ALP, ALT, AST and the De Ritis ratio and individual cancers and specifically whether even more moderate elevations of ALP predict an increased risk for future lung cancer. Verification of our results on a composite score of liver enzymes and its association with all-cause and cause-specific mortality are additionally warranted. If our results on associations of liver enzymes and mortality can be replicated in independent studies, the need to keep liver enzymes in the normal range would become even more urgent and monitoring of liver enzymes would harbour a preventive potential beyond liver diseases.

Acknowledgements The authors are grateful for the continuous participation of our cohort participants—-without their commitment this work would not have been possible. They thank Sandra Gonzalez Maldonado for her graphical support.

Contributors VK, DS, TK and RK contributed to the conception and design of the study. TJ organised the sample handling. VK, DS and AK conducted statistical analyses. VK wrote the initial manuscript. All authors reviewed and revised the manuscript. All authors read and approved the final manuscript.

Funding The present project was supported by the Helmholtz Association (Portfolio Theme "Metabolic Dysfunction") and the German Federal Ministry of Education and Research (BMBF) (Grant number 01ER0809) and cofunded by the German Center for Lung Research (DZL) and by the Helmholtz Association's Initiative on Aging and Metabolic Programming (AMPro).

Disclaimer None of the funders had any role in the study design; in collection, analysis, and interpretation of data; in the writing of the report; and in the decision to submit the manuscript for publication.

Competing interests None declared.

Patient consent for publication Not required.

Ethics approval The Medical Ethics Committee of the Heidelberg University (GEK 13/94) and the International Agency for Research on Cancer approved the study.

Provenance and peer review Not commissioned; externally peer reviewed.

Data availability statement Data are available upon reasonable request. EPICHeidelberg was launched in the 1990s. Unlike in new studies that we run today, public access to data from the EPIC population was not part of the study protocol at that time. Thus, the data protection statement and informed consent of the EPIC participants do not cover the provision of data in public repositories. Nevertheless, we are open to providing our dataset upon request for (a) statistical validation by reviewers and (b) pooling projects under clearly defined and secure conditions and based on valid data transfer agreements.

Open access This is an open access article distributed in accordance with the Creative Commons Attribution Non Commercial (CC BY-NC 4.0) license, which permits others to distribute, remix, adapt, build upon this work non-commercially, and license their derivative works on different terms, provided the original work is properly cited, appropriate credit is given, any changes made indicated, and the use is non-commercial. See: http://creativecommons.org/licenses/by-nc/4.0/.

ORCID iD

Verena Katzke http://orcid.org/0000-0002-6509-6555

\section{REFERENCES}

1 Dufour R, Qazi N. Evaluation of Liver Disease. In: Wagener G, ed. Liver anesthesiology and critical care medicine. New York: NY: Springer, 2012: 51-8.

2 Botros M, Sikaris KA. The de ritis ratio: the test of time. Clin Biochem Rev 2013;34:117-30.

3 Kunutsor SK, Apekey TA, Walley J. Liver aminotransferases and risk of incident type 2 diabetes: a systematic review and meta-analysis. Am J Epidemiol 2013;178:159-71.

4 Fraser A, Harris R, Sattar N, et al. Alanine aminotransferase, gammaglutamyltransferase, and incident diabetes: the British women's heart and health study and meta-analysis. Diabetes Care 2009;32:741-50.

5 Kühn T, Nonnenmacher T, Sookthai D, et al. Anthropometric and blood parameters for the prediction of NAFLD among overweight and obese adults. BMC Gastroenterol 2018;18:113.

6 Kunutsor SK, Apekey TA, Khan H. Liver enzymes and risk of cardiovascular disease in the general population: a meta-analysis of prospective cohort studies. Atherosclerosis 2014;236:7-17.

7 Choi KM, Han K, Park S, et al. Implication of liver enzymes on incident cardiovascular diseases and mortality: a nationwide population-based cohort study. Sci Rep 2018;8:3764.

8 Yi S-W, Lee S-H, Hwang H-J, et al. Gamma-Glutamyltransferase and cardiovascular mortality in Korean adults: a cohort study. Atherosclerosis 2017;265:102-9.

9 Kunutsor SK, Bakker SJL, Kootstra-Ros JE, et al. Inverse linear associations between liver aminotransferases and incident cardiovascular disease risk: the PREVEND study. Atherosclerosis 2015;243:138-47.

10 Wannamethee SG, Sattar N, Papcosta O, et al. Alkaline phosphatase, serum phosphate, and incident cardiovascular disease and total mortality in older men. Arterioscler Thromb Vasc Biol 2013;33:1070-6.

11 Koehler EM, Sanna D, Hansen BE, et al. Serum liver enzymes are associated with all-cause mortality in an elderly population. Liver Int 2014;34:296-304.

12 Wang J, Zhang D, Huang R, et al. Gamma-Glutamyltransferase and risk of cardiovascular mortality: a dose-response meta-analysis of prospective cohort studies. PLoS One 2017;12:e0172631.

13 Kunutsor SK, Apekey TA, Seddoh D, et al. Liver enzymes and risk of all-cause mortality in general populations: a systematic review and meta-analysis. Int J Epidemiol 2014;43:187-201.

14 Liu Z, Ning H, Que S, et al. Complex association between alanine aminotransferase activity and mortality in general population: a systematic review and meta-analysis of prospective studies. PLoS One 2014;9:e91410.

15 Long Y, Zeng F, Shi J, et al. Gamma-Glutamyltransferase predicts increased risk of mortality: a systematic review and meta-analysis of prospective observational studies. Free Radic Res 2014;48:716-28.

16 Kunutsor SK, Apekey TA, Van Hemelrijck M, et al. Gamma glutamyltransferase, alanine aminotransferase and risk of cancer: systematic review and meta-analysis. Int $\mathrm{J}$ Cancer 2015;136:1162-70.

17 Mok Y, Son D-K, Yun YD, et al. $\gamma$-Glutamyltransferase and cancer risk: the Korean cancer prevention study. Int J Cancer 2016;138:311-9.

18 Preyer O, Johansen D, Holly J, et al. $\gamma$-Glutamyltransferase and Breast Cancer Risk Beyond Alcohol Consumption and Other Life Style Factors - A Pooled Cohort Analysis. PLoS One 2016;11:e0149122.

19 Shackshaft L, Van Hemelrijck M, Garmo H, et al. Circulating gammaglutamyl transferase and development of specific breast cancer subtypes: findings from the apolipoprotein mortality risk (AMORIS) cohort. Breast Cancer Res 2017;19:22.

20 Yokoyama M, Watanabe T, Otaki Y, et al. Association of the aspartate aminotransferase to alanine aminotransferase ratio with BNP level and cardiovascular mortality in the general population: the Yamagata study 10-year follow-up. Dis Markers 2016;2016:4857917

21 Riboli E, Hunt KJ, Slimani N, et al. European prospective investigation into cancer and nutrition (EPIC): study populations and data collection. Public Health Nutr 2002;5:1113-24. 
22 Barlow WE, Ichikawa L, Rosner D, et al. Analysis of case-cohort designs. J Clin Epidemiol 1999;52:1165-72.

23 Scandinavian Health Ltd (SHL). Laboratoriumbepalingen. Available: https://www.shl-groep.nl/huisarts/laboratoriumbepalingen/ [Accessed 29 Oct 2018].

24 De Ritis F, Coltorti M, Giusti G. An enzymic test for the diagnosis of viral hepatitis; the transaminase serum activities. Clin Chim Acta 1957;2:70-4.

25 Prentice RL. A case-cohort design for epidemiologic cohort studies and disease prevention trials. Biometrika 1986;73:1-11.

26 Xue X, Xie X, Gunter M, et al. Testing the proportional hazards assumption in case-cohort analysis. BMC Med Res Methodol 2013;13:88

27 Mahady SE, Wong G, Turner RM, et al. Elevated liver enzymes and mortality in older individuals: a prospective cohort study. J Clin Gastroenterol 2017;51:439-45.

$28 \mathrm{Kim} \mathrm{K-N}$, Joo J, Sung HK, et al. Associations of serum liver enzyme levels and their changes over time with all-cause and cause-specific mortality in the general population: a large-scale National health screening cohort study. BMJ Open 2019;9:e026965.
29 Adams LA, Anstee QM, Tilg H, et al. Non-Alcoholic fatty liver disease and its relationship with cardiovascular disease and other extrahepatic diseases. Gut 2017;66:1138-53.

30 Targher G, Byrne CD, Lonardo A, et al. Non-Alcoholic fatty liver disease and risk of incident cardiovascular disease: a meta-analysis. $J$ Hepatol 2016;65:589-600.

31 Emdin M, Pompella A, Paolicchi A. Gamma-Glutamyltransferase, atherosclerosis, and cardiovascular disease: triggering oxidative stress within the plaque. Circulation 2005;112:2078-80.

32 Schoppet M, Shanahan CM. Role for alkaline phosphatase as an inducer of vascular calcification in renal failure? Kidney Int 2008;73:989-91.

33 Huang J, Gu T, Ying J. A meta-analysis survey of appropriate bone turnover markers in the detection of bone metastasis in lung cancer. Int J Clin Oncol 2017:22:1015-25.

34 Zhou Y, Yu Q-F, Peng A-F, et al. The risk factors of bone metastases in patients with lung cancer. Sci Rep 2017;7:8970.

35 Ruhl CE, Everhart JE. Diurnal variation in serum alanine aminotransferase activity in the US population. J Clin Gastroenterol 2013;47:165-73. 